\title{
Parathyroid hormone changes following denosumab treatment in postmenopausal osteoporosis
}

Polyzois Makras $^{1}$, Stergios A. Polyzos ${ }^{2}$, Athanasios Papatheodorou ${ }^{3}$, Panagiotis Kokkoris ${ }^{1,3}$, Daniel Chatzifotiadis ${ }^{4}$, and Athanasios D. Anastasilakis ${ }^{5}$

${ }^{1}$ Department of Endocrinology and Diabetes, 251 Hellenic Air Force \& VA General Hospital, Athens, Greece; ${ }^{2}$ Second Medical Clinic, Aristotle University of Thessaloniki, Ippokration Hospital, Thessaloniki, Greece; ${ }^{3}$ Department of Medical Research, 251 Hellenic Air Force and VA General Hospital, Athens, Greece; ${ }^{4}$ Division of Nuclear Medicine, 251 Hellenic Air Force and VA General Hospital, Athens, Greece; ${ }^{5}$ Department of Endocrinology, 424 Military Hospital, Thessaloniki, Greece.

Introduction: Denosumab is a fully human monoclonal antibody to RANKL and represents a distinct class of antiresorptives in osteoporosis treatment, since it inhibits osteoclast maturation in the early stages of development and osteoclast activity, rather than impairing viability of osteoclasts. In both preclinical and clinical studies denosumab induced a dose-dependent increase in parathyroid hormone (PTH) levels. This increase in PTH is considered compensatory against the transient dose-dependent decrease in serum calcium levels, while hypocalcemic events seem infrequent even among subjects not receiving calcium and/or vitamin $D(C a / D)$ supplements.

In this study we monitored PTH changes following a single injection of denosumab. The primary end point was the alteration of PTH 1 month after the injection while receiving a commonly used (1 gr/800 IU) or double-dose ( $2 \mathrm{gr} / 1,600$ IU) supplementation with calcium and vitamin $D$. The secondary end point was the alteration of PTH 6 months after denosumab injection.

\section{Methods:}

Design: Prospective, multicenter, study among postmenopausal women followed for 6 months.

Patients: 47 postmenopausal women followed in 2 outpatient clinics, requiring onset or continuation of osteoporosis treatment. We administered 1 gr calcium carbonate and 800 IU cholecalciferol daily for 6 months (Group A) or the double dose for the first month followed by the $1 \mathrm{gr} / 800 \mathrm{IU} \mathrm{Ca} / \mathrm{D}$ regimen for the next 5 months (Group B) (Figure 1).

Measurements: PTH alterations between and within groups, and their associations with serum $\mathrm{Ca}$ and bone markers.

Figure 3: Alterations of PTH, Ca, P1NP and CTX levels at month 1.

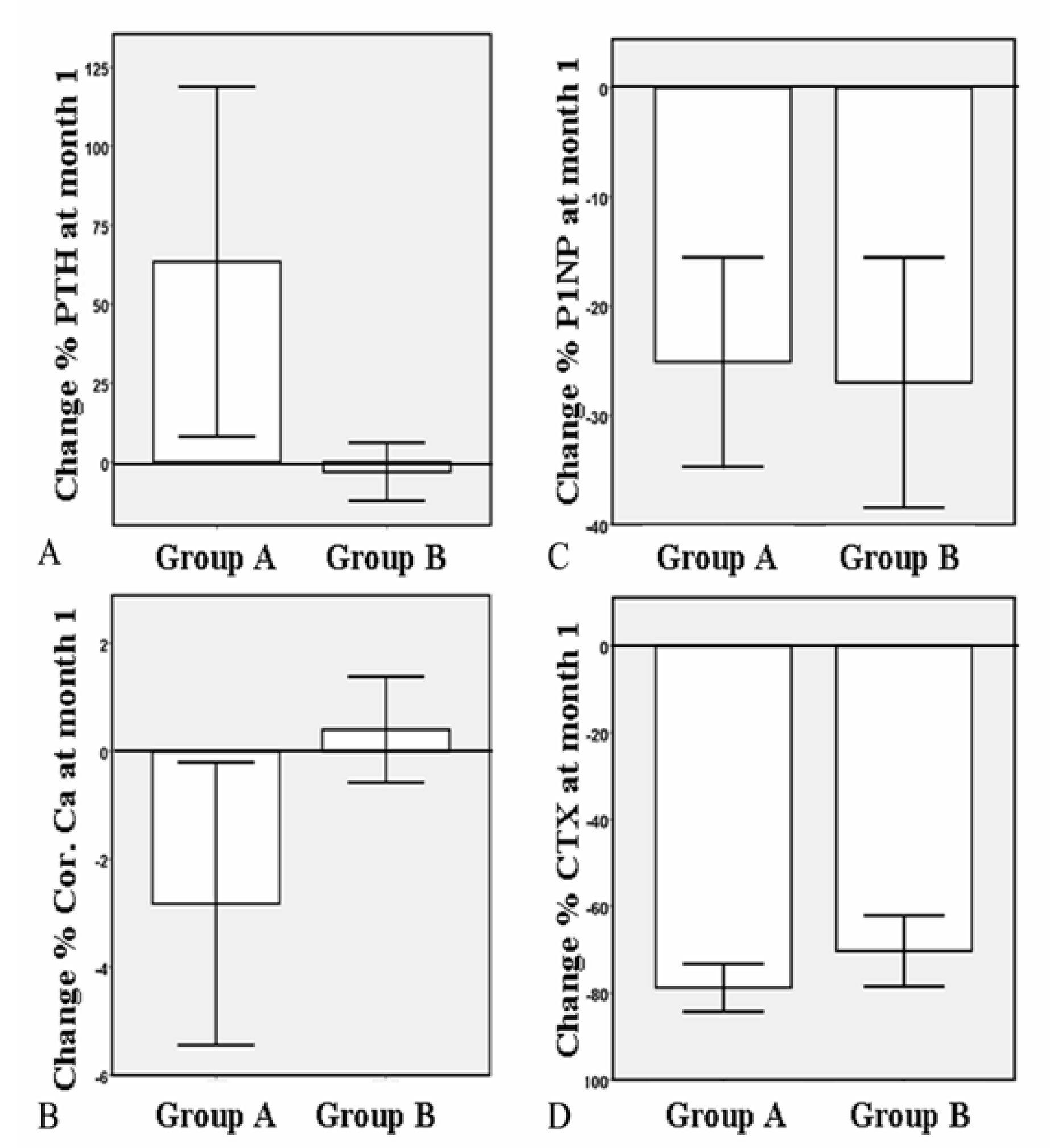

Figure 1: Calcium and cholecalciferol administration

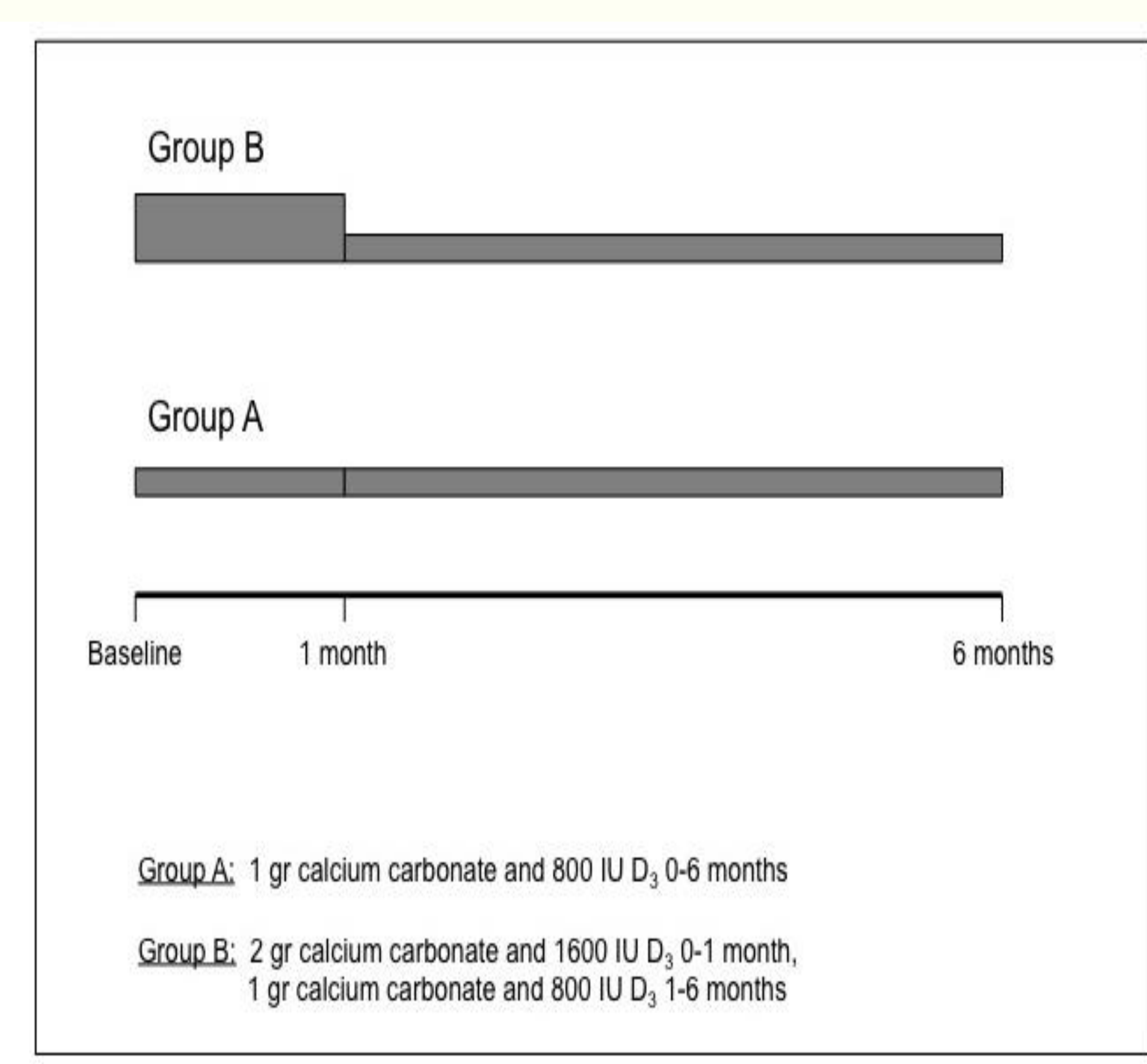

Figure 2: Previous treatment

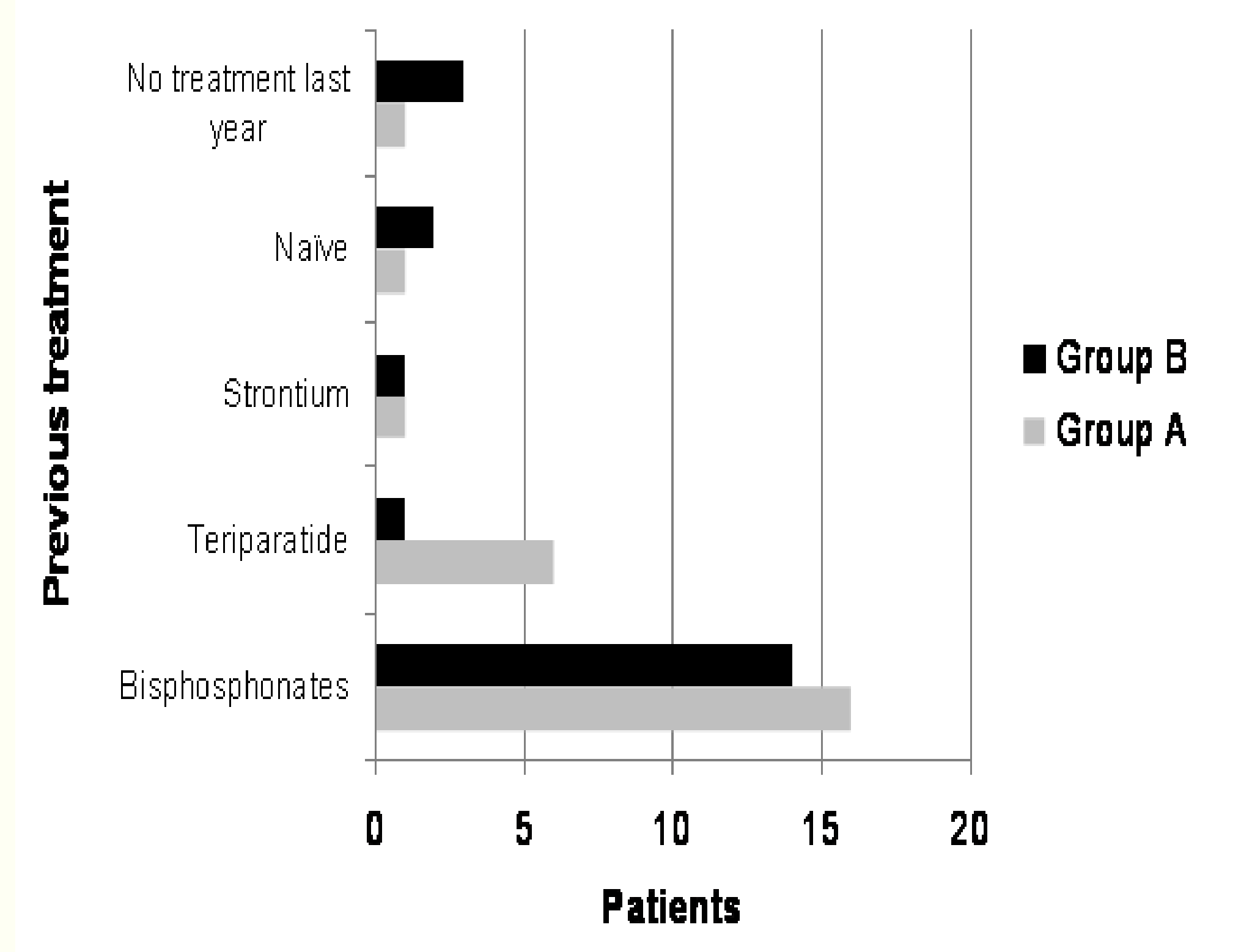

Table 1. Clinical, demographic and Bone Mineral Density data of both groups at baseline

\begin{tabular}{lccl}
\hline Variable & Group A & Group B & P.value \\
\hline Age (years) & $67.9 \pm 1.9$ & $67.3 \pm 2.4$ & 0.843 \\
BMI $\left(\mathrm{kg} / \mathrm{m}^{2}\right)$ & $26.2 \pm 0.9$ & $29.4 \pm 1.1$ & 0.067 \\
BMD Lumbar spine $\left(\mathrm{g} / \mathrm{cm}^{2}\right)$ & $0.828 \pm 0.018$ & $0.873 \pm 0.015$ & 0.066 \\
BMD femoral neck $\left(\mathrm{g} / \mathrm{cm}^{2}\right)$ & $0.689 \pm 0.020$ & $0.688 \pm 0.017$ & 0.97 \\
BMD Lumbar spine $(T-$-score $)$ & $-2.8 \pm 0.1$ & $-2.3 \pm 0.11$ & 0.009 \\
BMD femoral neck (T-score) & $-2.4 \pm 0.2$ & $-2.6 \pm 0.1$ & 0.514
\end{tabular}

BMI, Body Mass Index; BMD, Bone Mineral Density

\begin{tabular}{|c|c|c|c|c|c|c|c|}
\hline Variable & Group & Baseline & Month 1 & $\begin{array}{l}P \text {-value } \\
\text { between } \\
\text { baseline } \\
\text { and } \\
\text { month 1 }\end{array}$ & Month 6 & $\begin{array}{l}P \text {-value } \\
\text { between } \\
\text { month } 1 \\
\text { and } 6\end{array}$ & $\begin{array}{l}P \text {-value } \\
\text { between } \\
\text { baseline } \\
\text { and } \\
\text { month } 6\end{array}$ \\
\hline PTH $(\mathrm{pg} / \mathrm{mL})$ & A & $34.8 \pm 2.8$ & $62 \cdot 4 \pm 13 \cdot 3$ & 0.026 & $40 \cdot 7 \pm 4 \cdot 0$ & 0.532 & 0.029 \\
\hline$P$-value between groups & B & $\begin{array}{l}41 \cdot 7 \pm 2.6 \\
0.079\end{array}$ & $\begin{array}{r}39 \cdot 8 \pm 2 \cdot 8 \\
0.982\end{array}$ & 0.263 & $\begin{array}{r}44 \cdot 2 \\
0.553\end{array}$ & 0.638 & 0.567 \\
\hline $25(\mathrm{OH})$ vitamin $\mathrm{D}(\mathrm{ng} / \mathrm{mL})$ & $\mathrm{A}$ & $26.9 \pm 2.6$ & $28 \cdot 4 \pm 2 \cdot 7$ & 0.590 & $36 \cdot 6 \pm 4 \cdot 1$ & 0.009 & 0.016 \\
\hline$P_{\text {-value between arounc }}$ & B & $\begin{array}{r}27 \cdot 2 \pm 2 \cdot 0 \\
0.993\end{array}$ & $\begin{array}{r}28.4 \pm 1.7 \\
0.989\end{array}$ & 0.464 & $\begin{array}{r}35 \cdot 7 \pm 2 \cdot 6 \\
0.888\end{array}$ & 0.004 & 0.002 \\
\hline $\begin{array}{l}\text { P-value between groups } \\
\text { PINP (ng/mL) }\end{array}$ & & & & & 0.848 & & \\
\hline 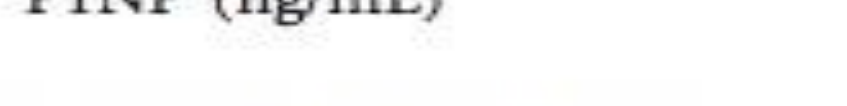 & B & $\begin{array}{l}49.1 \pm 8.2 \\
37.4 \pm 6.0\end{array}$ & $\begin{array}{l}35.6 \pm 4.1 \\
26.4 \pm 5.8\end{array}$ & 0.001 & $\begin{array}{l}16.0 \pm 2.0 \\
17.8 \pm 3 \cdot 1\end{array}$ & $\begin{array}{l}0.04 \\
0.003\end{array}$ & $<0.001$ \\
\hline$P$-value between groups & & 0.144 & 0.020 & & 0.875 & & \\
\hline $\mathrm{CTX}(\mathrm{ng} / \mathrm{mL})$ & $\underset{B}{A}$ & $\begin{array}{l}0.226 \pm 0.045 \\
0.223 \pm 0.040\end{array}$ & $\begin{array}{l}0.035 \pm 0.003 \\
0.054 \pm 0.010\end{array}$ & $\begin{array}{l}<0.001 \\
<0.001\end{array}$ & $\begin{array}{l}0.105 \pm 0.015 \\
0.112 \pm 0.015\end{array}$ & $<0.001$ & . \\
\hline$P$-value between groups & & $0-894$ & 0.240 & & 0.773 & & \\
\hline Corrected calcium (mg/dL) & A & $9.5 \pm 0.1$ & $9 \cdot 3 \pm 0 \cdot 1$ & 0.031 & $9 \cdot 6 \pm 0.1$ & 0.003 & 0.758 \\
\hline$P$-value between groups & в & $9.3 \pm 0.1$ & $\begin{array}{r}9.4 \pm 0.1 \\
0.524\end{array}$ & 0.469 & $\begin{array}{r}9.4 \\
0.370\end{array}$ & 0.383 & 0.127 \\
\hline Phosphate (mg/dL) & A & $3.7 \pm 0.1$ & $3 \cdot 5 \pm 0.1$ & 0.136 & $3 \cdot 6 \pm 0 \cdot 1$ & 0.882 & 0.131 \\
\hline & B & $3 \cdot 8 \pm 0.1$ & $3 \cdot 8 \pm 0.1$ & 0.894 & $3 \cdot 7 \pm 0 \cdot 1$ & 0.392 & 0.496 \\
\hline$P$-value between groups & & 0.524 & 0.061 & & 0.554 & & \\
\hline $\mathrm{eGFR}\left(\mathrm{mL} / \mathrm{min} / 1 \cdot 73 \mathrm{~m}^{2}\right)$ & A & 73.6 73.0 & $70.4 \pm 3.4$ & 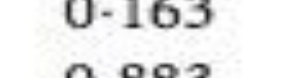 & $69.3 \pm 3.4$ & 0.362 & 0.117 \\
\hline$P$-value between groups & & 0.953 & 0.582 & & & & 0.731 \\
\hline
\end{tabular}

Results: There were no between group differences regarding previous treatment $(p=0.325)$ (Figure 2) or regarding previous bisphosphonate use $(p=0.820)$. Eight patients had previously experienced one, two patients had two, and one patient had three lowenergy fractures $(p=0.258$ for between group fractures at baseline). No significant differences were found at baseline regarding BMD and biochemistry (Tables 1 and 2).

Regarding between group differences (Group A vs. Group B), month 1 and month 6 comparative data were similar, except for P1NP, which was significantly higher in Group A than $B$ at month 1 (Table 2). However, the mean percent change between month 1 and baseline for PTH $\left[\Delta\left(\mathbf{P T H}_{1-0}\right)\right]$ was significantly higher in Group $A$ than $B(63.5 \%$ $\pm 28.2 \%$ vs. $-3.0 \% \pm 4.7 \%, p=0.029)$, whereas $\Delta\left(\right.$ Corrected calcium $\left._{1-0}\right)$ was significantly lower in Group A than B $(-2.8 \% \pm 1.3 \%$ vs. $0.4 \% \pm 0.5 \%$, $\mathbf{p}=\mathbf{0 . 0 3 1})$; there were no significant changes in $\Delta\left(\mathbf{P 1 N P}_{1-0}\right)$ and $\Delta\left(\mathrm{CTX}_{1-0}\right)($ Figure 3$)$.

Regarding within group differences, PTH levels were significantly higher at month 1 and 6 in $A$, but not in Group B (Table 1). Corrected calcium levels were significantly decreased in Group A, but not in Group B, at month 1 and returned to baseline values at month 6 (Table 2). Phosphate levels were not significantly changed in either group.

$\Delta\left(\mathrm{PTH}_{1-0}\right)$ was significantly inversely correlated with $\Delta\left(\right.$ corrected calcium $\left.{ }_{1-0}\right) \quad\left(r_{s}=-0.610 ; p=0.002\right)$, and $\Delta\left(\mathrm{CTX}_{1-0}\right)\left(\mathrm{r}_{\mathrm{s}}=\mathbf{- 0 . 6 9 7} ; \mathrm{p}=\mathbf{0 . 0 0 3}\right)$ in Group $\mathrm{A}$, but not in Group $B\left(r_{s}=\mathbf{- 0 . 1 8 1} ; p=0.433, r_{s}=\mathbf{- 0 . 0 5 2} ; p=0.823\right.$, $r_{s}=-0.30 ; p=0.893$, respectively).

No adverse event, including hypo- or hypercalcaemia and hypercalciuria, was recorded throughout the study.

Conclusion: Calcium and vit. D supplementation at a dose of $2 \mathrm{gr} / 1,600$ IU, but not $1 \mathrm{gr} / 800$ IU, attenuated the decrease in serum $\mathrm{Ca}$ and the compensatory increase in PTH following a single s.c. injection of denosumab 60mg. Therefore, an increase of PTH should be expected, at least following the first administration of denosumab in common clinical practice. The effect of this compensatory consequence in bone metabolism warrants further investigation. 